\title{
Intrinsic in-plane nodal chain and generalized quaternion charge protected nodal link in photonics
}

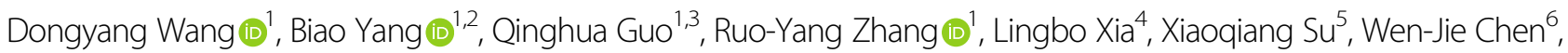 \\ Jiaguang Han (1) $^{7}$, Shuang Zhang ${ }^{8}$ and C. T. Chan (1)
}

\begin{abstract}
Nodal lines are degeneracies formed by crossing bands in three-dimensional momentum space. Interestingly, these degenerate lines can chain together via touching points and manifest as nodal chains. These nodal chains are usually embedded in two orthogonal planes and protected by the corresponding mirror symmetries. Here, we propose and demonstrate an in-plane nodal chain in photonics, where all chained nodal lines coexist in a single mirror plane instead of two orthogonal ones. The chain point is stabilized by the intrinsic symmetry that is specific to electromagnetic waves at the $\Gamma$ point of zero frequency. By adding another mirror plane, we find a nodal ring that is constructed by two higher bands and links with the in-plane nodal chain. The nodal link in momentum space exhibits non-Abelian characteristics on a $C_{2} T$ - invariant plane, where admissible transitions of the nodal link structure are determined by generalized quaternion charges. Through near-field scanning measurements of bi-anisotropic metamaterials, we experimentally mapped out the in-plane nodal chain and nodal link in such systems.
\end{abstract}

\section{Introduction}

Topological photonics has attracted a lot of attention recently ${ }^{1,2}$. The application of topological band theory to photonics not only opens the door to novel devices such as topological lasers ${ }^{3-7}$, but also stimulates the exploration of new topological phases, such as Floquet ${ }^{8}$ and highorder topological insulators ${ }^{2,9}$. Photonic systems offer a mature and highly flexible platform for topological phase discovery and realization ${ }^{10-28}$. We know that most electronic topological systems have their photonic counterparts, except for those depending on the intrinsic properties of fermion system, for example, 2D and 3D topological insulators ${ }^{29,30}$ with $T^{2}=-1$, where $T$ is the

\footnotetext{
Correspondence: Jiaguang Han (jiaghan@tju.edu.cn) or Shuang Zhang (s.zhang@bham.ac.uk) or C. T. Chan (phchan@ust.hk)

${ }^{1}$ Department of Physics, Hong Kong University of Science and Technology, Hong Kong, China

${ }^{2}$ College of Advanced Interdisciplinary Studies, National University of Defense Technology, Changsha, China

Full list of author information is available at the end of the article

These authors contributed equally: Dongyang Wang, Biao Yang, Qinghua Guo
}

time reversal operator. On the other hand, there are symmetries that are unique to electromagnetic (EM) waves, which can intrinsically protect the band degeneracies at isolated points in the momentum space ${ }^{31-33}$. Topological systems realized using such symmetries are uniquely "photonic", having no counterparts in electronic or phononic systems.

Identifying nodal features in the band structure of topological materials, such as nodal points (Dirac or Weyl points) or nodal lines, can help to understand their topological characters. Among various topological features in momentum space, nodal chain ${ }^{34-38}$ is a special configuration of nodal lines ${ }^{35,39-53}$ where two nodal curves touch at isolated points. It is generally perceived that the two nodal lines should reside on two separate mirror planes, each protected by their corresponding mirror symmetries. The chain points are then found to be stabilized on their intersection lines. Here, making use of symmetries being intrinsic to electromagnetism, we theoretically propose and experimentally demonstrate a type

\section{(c) The Author(s) 2021}

(c) (i) Open Access This article is licensed under a Creative Commons Attribution 4.0 International License, which permits use, sharing, adaptation, distribution and reproduction c. in any medium or format, as long as you give appropriate credit to the original author(s) and the source, provide a link to the Creative Commons license, and indicate if changes were made. The images or other third party material in this article are included in the article's Creative Commons license, unless indicated otherwise in a credit line to the material. If material is not included in the article's Creative Commons license and your intended use is not permitted by statutory regulation or exceeds the permitted use, you will need to obtain permission directly from the copyright holder. To view a copy of this license, visit http://creativecommons.org/licenses/by/4.0/. 


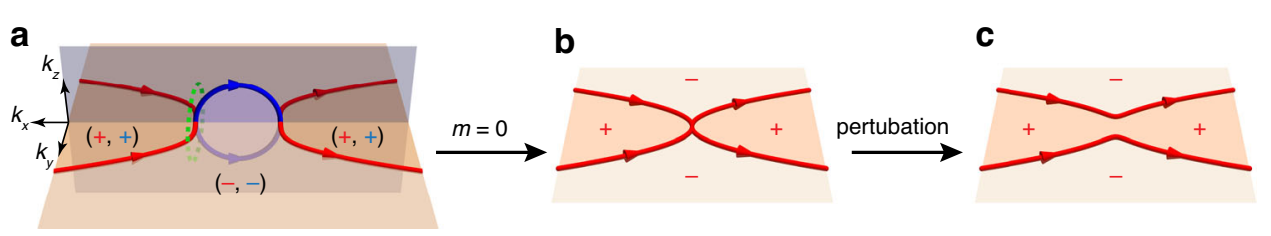

Fig. 1 Transition from orthogonal nodal chain to in-plane nodal chain and separated nodal lines. a A normal nodal chain embedded in two orthogonal mirror planes. Green circle indicates the $\pi_{1}$ homotopy loop. b The blue nodal ring can be shrunk continuously by tuning the system parameter $m$ (see text), resulting in an in-plane nodal chain when $m=0$. c Further perturbation finally gaps the chain point. For all panels, " \pm " indicates the signs of mirror symmetries eigenvalues, red color for $M_{z}$ and blue color for $M_{y}$. Within the framework of the two-band model, the eigenstates bounded inside the red (blue) degeneracy lines are of even (odd) parity, those outside are of odd (even) parity. Arrows indicate orientations of nodal lines with $\mathbb{Z}$ classified topological charges in a real two-band model

of in-plane nodal chain, where the nodal lines are chained together in a single plane. In-plane chain points are usually fragile against perturbations, but the chain point in our system is protected by the electromagnetic intrinsic symmetry at the $\Gamma$ point unless a cut-off frequency is introduced due to artificial resonances. The in-plane nodal chain is uniquely stable in photonics due to the internal symmetries of the Maxwell equations and has no counterparts in other systems. This new type of nodal chain should widely exist in photonic systems, as long as additional symmetries such as mirror symmetries, protect the existence of nodal line branches. We also find a nodal ring linking with the in-plane nodal chain in our studied model with only two mirror symmetries, and the linked nodal structure exhibits non-Abelian features ${ }^{54-59}$ on a $C_{2} T$ - invariant plane ${ }^{57}$. The present study provides a realization of non-Abelian nodal links in the absence of Parity-Time (PT) symmetry ${ }^{58}$, and can be described using generalized quaternion charges of the photonic multiband topology $y^{54,55}$. Finally, we experimentally demonstrate the in-plane nodal chain and nodal link with bianisotropic metamaterials.

\section{Results}

We first use a generic two-band Hamiltonian to show the presence of the in-plane nodal chain. We then show that photonic intrinsic symmetry can stabilize the chain point. The Hamiltonian that exhibits the nodal line consists of two Pauli matrices as,

$$
H=d_{x} \sigma_{x}+d_{z} \sigma_{z}
$$

where $d_{x}=k_{y} k_{z}$, and

$$
d_{z}=\left(k_{x}^{2}-k_{y}^{4}-m\right)+\left(k_{x}^{2}+k_{z}^{2}-m\right)
$$

The nodal line structure, as a function of the parameter $m$, is shown in Fig. 1. For a value of $m=+1$, there are two chain points as shown in Fig. 1a. Going from left to right across the chain point, the two mirror-symmetry (both are represented by $\left.\sigma_{z}\right)$ eigenvalues change from $\left(M_{z}, M_{y}\right)=$
$(+1,+1)$ to $(-1,-1)$ on the intersection line of the two mirror planes $\left(k_{y}=k_{z}=0\right)$. For a two-band model with PT symmetry, the eigenfunctions are purely real, so that a loop encircling the chain point (or a general point on the nodal line) carries a $\mathbb{Z}$-valued charge, i.e., integer charges with positive or negative signs, which can be understood as the orientation defined on each nodal line ${ }^{54}$. Keeping the mirror symmetries intact, one can smoothly eliminate the vertical nodal ring (the blue one) by tuning $m$ to zero, as shown in Fig. 1b, leading to an in-plane nodal chain. Meanwhile, the two out-of-plane chain points vanish concomitantly and merge into an in-plane chain point. The mirror eigenvalues of the eigenstates on the $k_{y}=k_{z}=$ 0 line have the same $(+)$ sign across the chain point, which indicate that the in-plane chain point is no longer stable since the symmetry eigenvalue sign change going across that point no longer exists. As shown in Fig. 1c, the in-plane chain point can be gapped by tuning $m$ to negative values while preserving the mirror symmetry eigenvalues as labelled.

In photonic systems, however, the in-plane nodal chain can be stabilized by the intrinsic symmetry of electromagnetic wave at the $\Gamma$ point of zero frequency. In order to demonstrate the stable in-plane chain point, we start with an effective model of bi-anisotropic material, where inversion symmetry is explicitly broken, but two mirror symmetries preserve the nodal lines, one for the in-plane nodal chain and the other for the nodal ring that contributes to the nodal link. The effective constitutive parameters for the bi-anisotropic metamaterial under consideration (explicit design and experimental realization will be discussed in subsequent sections) are:

$$
\vec{\varepsilon}=\left[\begin{array}{ccc}
\varepsilon & 0 & 0 \\
0 & \varepsilon & 0 \\
0 & 0 & \varepsilon_{b}
\end{array}\right], \vec{\mu}=\left[\begin{array}{ccc}
1 & 0 & 0 \\
0 & 1 & 0 \\
0 & 0 & \mu
\end{array}\right], \vec{\varsigma}=\left[\begin{array}{ccc}
0 & 0 & 0 \\
0 & 0 & 0 \\
-i \chi & -i X & 0
\end{array}\right], \vec{\xi}=\left[\begin{array}{ccc}
0 & 0 & i \chi \\
0 & 0 & i X \\
0 & 0 & 0
\end{array}\right] .
$$

The matrix elements depend on frequency and the structural parameters of the metamaterials, and take the form of $\varepsilon=\varepsilon_{b}+\frac{1}{\omega_{0}^{2}-\omega^{2}} \frac{l^{2}}{L}, \mu=1+\frac{2}{\omega_{0}^{2}-\omega^{2}} \frac{\omega^{2} A^{2}}{L}, X=\frac{\omega}{\omega_{0}^{2}-\omega^{2}} \frac{A l}{L}$, where $\omega_{0}$ is a structure-induced resonance frequency, $\varepsilon_{b}$ is the permittivity of substrate material, $L$ is the effective 

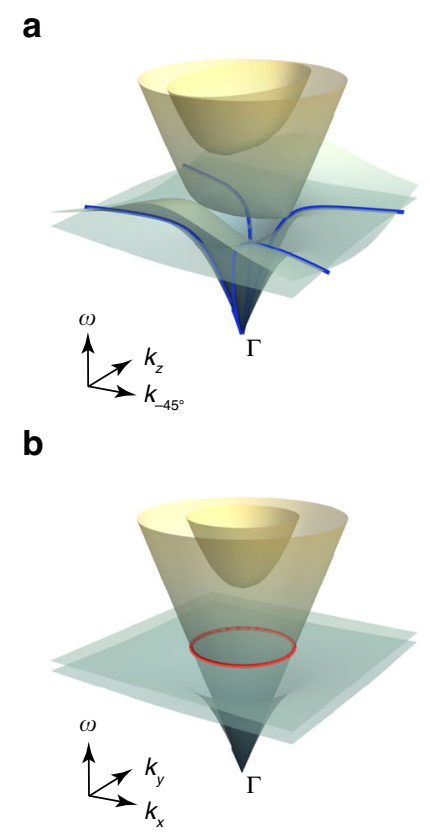

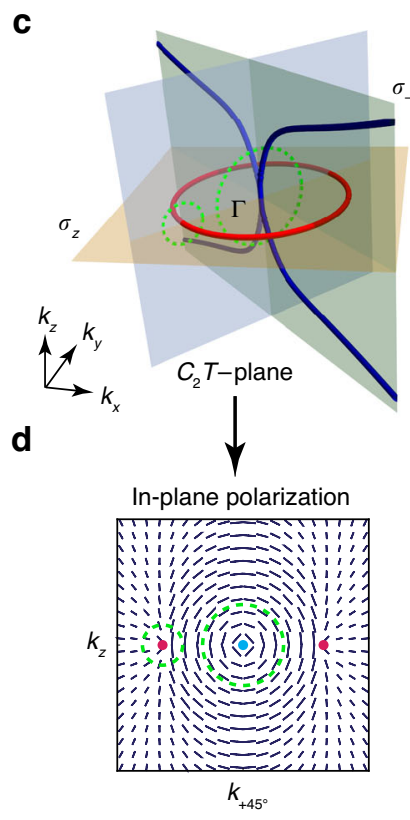

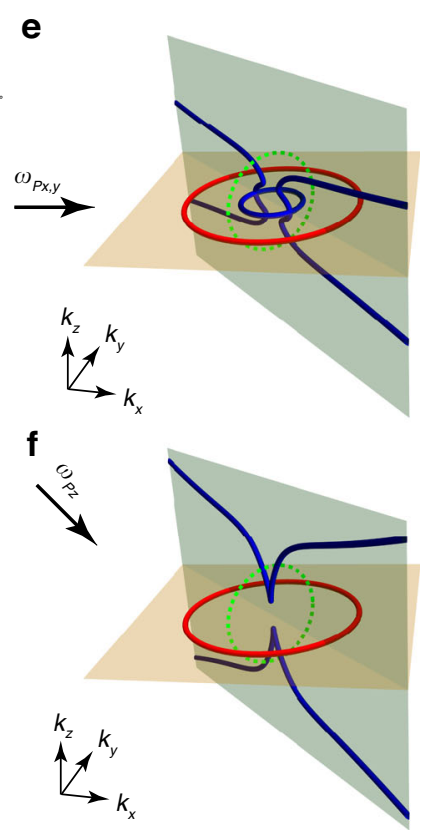

Fig. 2 In-plane nodal chain and nodal link in bi-anisotropic metamaterials. a Band structure of the bi-anisotropic effective medium model in the

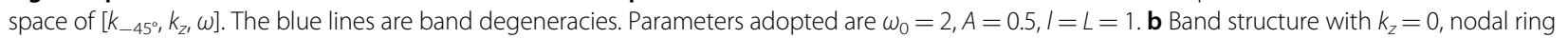
is shown in red. c Nodal link in momentum space, in-plane nodal chain is shown in blue and the red circle is a nodal ring. Green circles indicate the $\pi_{1}$ homotopy loops in the $C_{2} T$ - invariant plane. $\mathbf{d}$ The projected polarization states of the 2 nd band at the $C_{2} T$ - invariant plane, the red dots are cutpositions of the nodal ring, blue dot is the in-plane nodal chain point at the $\Gamma$ point. Green circles indicate $\pi_{1}$ loops encircling different degeneracy nodes as in c. e The breaking of chain point with an in-plane plasmonic resonance, where a new nodal ring (blue) emerges on the $k_{z}=0$ plane. The nodal ring connects the two nodal line branches as a consequence of the -1 non-Abelian charge accumulated along the green loop. $\mathbf{f}$ The breaking of chain point with $z$ direction plasmon resonance. No new nodal structure shows up since the green loop still encircles two nodal lines and the -1 charge remains conserved

inductance, $l$ and $A$ are the effective length and area of resonators. The bi-anisotropic metamaterial's response to electromagnetic waves is given by Maxwell equations as $\nabla \times \mathrm{E}=-\partial_{\mathrm{t}}(\vec{\mu} H+\overleftrightarrow{\varsigma} E), \nabla \times \mathrm{H}=\partial_{\mathrm{t}}(\vec{\varepsilon} \mathrm{E}+\vec{\xi} \mathrm{H})$.

The energy bands of the proposed bi-anisotropic material can be studied with these effective constitutive parameter matrices. In Fig. 2a, band dispersions are calculated on the diagonal mirror plane of $k_{x}=-k_{y}$, where degeneracy lines are found between the 1st and 2nd bands and are highlighted as blue curves. As frequency decreases, the equi-frequency surfaces shrink, and the degenerate lines move closer to each other which finally merge at zero frequency at the $\Gamma$ point. By showing the degeneracy lines in the full momentum space, we can see from Fig. $2 c$ that a pair of nodal lines (blue curves) touches each other at the $\Gamma$ point. These nodal lines are embedded in a single plane of mirror symmetry, and as such, they are concrete examples of in-plane nodal chains we have referred to. It is important to note that the chain point is robust to perturbations due to the stable degeneracy of electromagnetic wave at zero frequency.

By examining the band structure on the other mirror plane $\left(k_{z}=0\right)$, a degeneracy ring (marked in red) between the 2 nd and 3rd bands is found as shown in Fig. $2 b$. The nodal ring is induced by the electric resonance and appears at the frequency of $\left.\omega\right|_{\varepsilon=0}$, where a dispersionless longitudinal mode (flat band) appears and intersects the propagating transverse mode. More interestingly, the inplane nodal chain (blue) threads through the nodal ring (red) in the momentum space as shown in Fig. 2c. A nodal link is thus constructed by three adjacent bands which give enough freedom to define non-Abelian charges. The non-Abelian charges represent the frame rotations of a set of real eigenfunctions, which form the elements of nonAbelian (generalized) quaternion groups. The charges characterize the band degeneracies and explain the protection of the global nodal structure that will be discussed later. The peculiar nodal link in Fig. 2c can be analytically described with the effective medium model (supplementary information 3), and linear dispersions of the nodal structures can be checked by considering $k \cdot p$ expansions (Supplementary information 4).

The nodal link in momentum space can be characterized by non-Abelian topological charges. We consider the first homotopy group $\left(\pi_{1}\right)$ loop encircling the chain point in the $C_{2} T$ - invariant plane, as indicated in Fig. $2 \mathrm{c}$ with a green circle. In the $C_{2} T$ - invariant plane, the photonic bands have real eigenvectors, and at each $k$-point, the set 
of eigenvectors defines a frame which rotates as the $k$ point goes along the green-colored loop. Such an eigenvector frame rotation can be described using generalized quaternion charges $n_{\Gamma}$ ( $\Gamma$ indicating the $\pi_{1}$ loop), which extend the notion of quaternion charges to system with more than three bands and account for the multi-band topology $y^{54,55}$. Degeneracy between the $j$ th and $(j+1)$ th bands can be characterized as the non-Abelian charge of $n_{\Gamma}=g_{j}$ (see Supplementary information 5 for definition). In particular, frame rotation angles of 0 and $2 \pi$ are distinguished by $n_{\Gamma}=+1$ and -1 , with -1 being the nontrivial case. The non-Abelian topological charge of -1 is uniquely interesting as it describes a topological character of multiple bands that cannot be described using the Berry phase quantization of individual bands. Since the non-Abelian topological charges are closely related to the rotation of the eigen polarization states, we show the projected polarization states $\left(E_{+45^{\circ}}, E_{z}\right)$ of the 2nd band on the $C_{2} T$ - invariant plane in Fig. $2 \mathrm{~d}$, which illustrate the polarization rotation around the chain point (blue dot) as well as the nodal ring intersections in the $C_{2} T$ - invariant plane (red dots). As shown in Fig. 2d, a winding phase of $2 \pi$ is found for the larger green loop encircling the chain point (blue dot). Such a winding pattern is akin to the order-parameter field in the vicinity of a $2 \pi$ disclination line defect in biaxial nematic liquid crystals ${ }^{60,61}$, which can be characterized by a quaternion charge of -1 that describes $3 D$ rotations. The $2 \pi$ winding phase here indicates the generalized quaternion charge of $n_{\Gamma}=-1$, a generalization to higher dimension as we are considering 5 bands together (see detail of non-Abelian charges calculation in supplementary information 5). The non-trivial topological charge of $n_{\Gamma}=-1$ forbids the annihilation or breaking of the encircled nodal chain branches and plays an essential role in protecting the global nodal link structure in momentum space. The winding phase around the nodal ring intersection (red dots encircled by the small green circle in Fig. 2d) is found to be $\pi$, which corresponds to the generalized quaternion charge of $n_{\Gamma}=$ $\pm g_{2}$ that gives a characterization of a degeneracy node between the 2nd and 3rd bands.

The notion of non-Abelian charges can elegantly explain or predict admissible transitions of the nodal link as system parameter changes. The in-plane chain point at $\Gamma$ is stable against perturbations that do not introduce a cut-off frequency which gaps the $\Gamma$ point. In order to demonstrate the transition rule of the nodal link, we introduce plasmon resonances to the bi-anisotropic model, which gap the $\Gamma$ point, pushing the nodal lines away from zero frequency. Two configurations of artificial plasmon resonances are considered for illustration purpose, one with resonance directions aligned with $\mathrm{x}$ and $\mathrm{y}$ axes, and the other along the $\mathrm{z}$ direction. In both cases, two mirror symmetries are maintained so as to protect the nodal lines. In Fig. 2e, we show the nodal structures in momentum space with in-plane artificial resonances along the $\mathrm{x}$ and $\mathrm{y}$ directions. The artificial resonances introduce non-zero energy (or mass) to the Hamiltonian at the $\Gamma$ point. The chain point is now broken, however, in contrary to the two-band model in Fig. 1b, the nodal lines encircled by the green loop in Fig. 2e cannot just disappear, as a consequence of the conservation of -1 generalized quaternion charge. A new nodal ring (blue) has to emerge so as to conserve the charge of -1 and connects the two nodal line branches. In a different configuration, artificial resonance is introduced along the $k_{z}$ direction in Fig. 2f. The chain point is also broken by the resonance, but in contrary to the previous case, the nodal chain here is allowed to break along $k_{z}$ direction, and no new nodal structures are required to emerge. The transition mechanism can be understood from the schematic in Fig. 2f, where we can find that the two separated nodal line branches are still encircled by the green circle, and the -1 non-Abelian charge remains conserved. In addition, the protection by non-Abelian charges when plasmon resonance is introduced can again be explained by the rotation of eigen polarization states on the $C_{2} T$ - invariant plane (as in the case of Fig. 2d), and detail discussions are shown in supplementary information 6 .

Next, we demonstrate the proposed model experimentally in the microwave regime with a class of metallic metamaterials which exhibits topological line nodes ${ }^{58,62,63}$. A non-centrosymmetric meta-structure design is shown in Fig. 3a, where two planar split-ring triangular resonators are placed on the $\mathrm{z}=0$ mirror plane. Another mirror plane can be found along the diagonal direction as indicated by red dashed line. In the long wavelength limit, the metastructure exhibits bi-anisotropic couplings as described by the effective medium parameters shown above. The driving electric fields induce currents running along $x$ and $y$ directions, and the "split-ring" configuration results in a magnetic moment along the $z$ direction as schematically shown in Fig. 3a. In a reciprocal manner, the out of plane magnetic component $H_{z}$ of incoming electromagnetic wave will induce in-plane polarizations of $P_{x}$ and $P_{y}$ accordingly (supplementary information 1-2). The resonating units are arrayed periodically as shown in Fig. 3b. They collectively contribute to the bi-anisotropic response of the metamaterials.

By utilizing the CST microwave studio numerical package, photonic band dispersions of the metamaterial structure in Fig. 3a are calculated and shown in Fig. 3c. A degeneracy point is found along $\mathrm{M}^{\prime}$ - A direction (blue dot) embedded in the diagonal mirror plane, which originates from the in-plane nodal chain. Additional degeneracy points of a nodal ring can be found along $\Gamma-\mathrm{X} / \mathrm{M}^{\prime}$ directions (e.g., red dot) at $k_{z}=0$ plane. The linear band dispersions at these marked degenerate points 
a

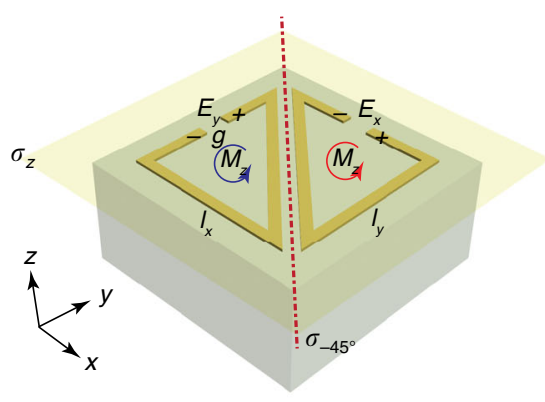

C

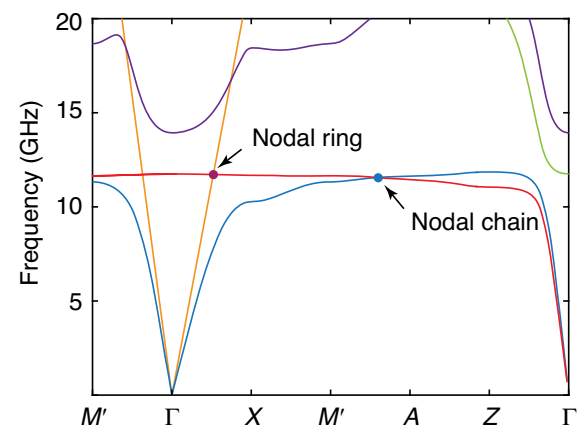

b

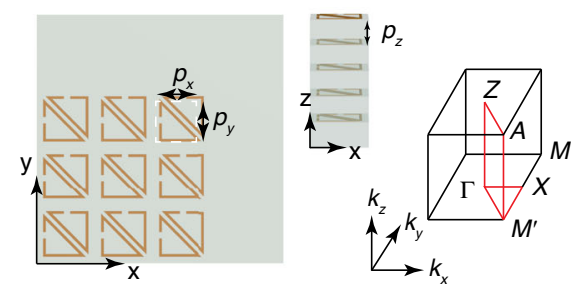

f
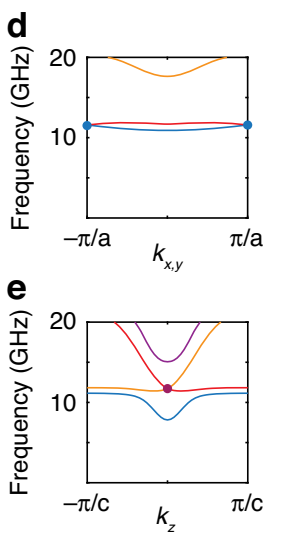

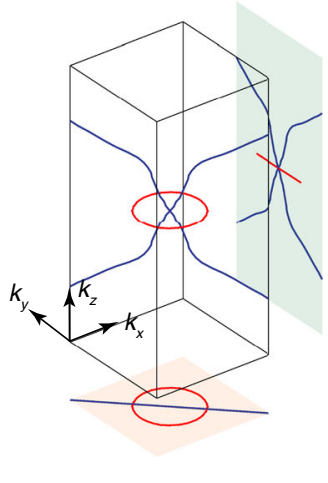

Fig. 3 Realistic bi-anisotropic metamaterials with in-plane nodal chain and nodal link. a Schematic of the bi-anisotropic metamaterial. Splitring resonators are arranged on the $\sigma_{z}$ mirror plane. The resonators also possess another mirror symmetry of $\sigma_{-45^{\circ}}$ as indicated by red dashed line. Side length of the resonator is $I_{x, y}=3.3 \mathrm{~mm}$, and resonance gap is $g=0.5 \mathrm{~mm}$. $\mathbf{b}$ The units are repeated to form 3D metamaterials, with lattice constants $p_{x}=4.5 \mathrm{~mm}, p_{y}=4.5 \mathrm{~mm}$ and $p_{z}=2 \mathrm{~mm}$. The Brillouin zone of the metamaterials is shown to the right. c Numerically calculated band structures, with degenerate points from in-plane nodal chain and nodal ring marked as blue and red dots, respectively. $\mathbf{d}$, e Linear band dispersions along $k_{x, y} / k_{z}$ directions around the blue / red dot marked in $\mathbf{c}$. $\mathbf{f}$ Nodal link in 3D momentum space corresponding to metamaterial shown in $\mathbf{b}$, and projections of the nodal link onto the $k_{y}-k_{z}$ (green) and $k_{x}-k_{y}$ (orange) planes

can be further examined along $k_{x, y}$ and $k_{z}$ directions, as shown in Fig. 3d, e (blue and red dots), respectively.

The nodal lines in momentum space formed by the intersections of the lowest three bands of the metastructure are shown in Fig. 3f, where the in-plane nodal chain (blue) and nodal ring (red) are found on two perpendicular mirror planes. The in-plane nodal chain is protected by the intrinsic symmetry at $\Gamma$ point and extends to Brillouin zone boundaries. The nodal chain threads through the nodal ring and a nodal link constructed by three adjacent bands is thus formed. The projections of the nodal lines on the $k_{x}-k_{y}$ plane (orange) and $k_{y}-k_{z}$ plane (green) are also shown in Fig. 3f.

We then fabricate the sample (80 stacked PCB layers along the $\mathrm{z}$ direction; $100 \times 10$ unit cells in the $\mathrm{xy}$ plane) and experimentally characterize these nodal lines. An experimental configuration is shown in Fig. 4a, where the side surface ( $x z$ plane) is configured for raster-scanning with a near-field probe antenna (red antenna). A point source (blue antenna) is placed at the corner of the sample and the field distribution on the side surface is to be measured. The measured field distributions can be subsequently Fourier transformed to obtain the projected band information in momentum space. The calculated inplane nodal chain corresponding to the experimental configuration is shown in the space of $\left[k_{x}, k_{z}, \omega\right]$ in Fig. $4 \mathrm{~b}$, which projects onto the $k_{x}-k_{z}$ plane as the purple curve. The equi-frequency contours (EFCs) intersect with the blue curves as four cyan points of bands degeneracies, as will be experimentally measured later. The in-plane nodal chain is also shown in 3D momentum space of Fig. 4c as blue lines.

In Fig. 4d, e, we show respectively the calculated and experimentally measured band spectra along the lines $\bar{\Gamma}$ $\overline{\mathrm{X}}-\overline{\mathrm{A}}-\bar{\Gamma}$ defined in Fig. $4 \mathrm{~b}$. A touching point of projected bands, marked with a blue dot, is observed along $\overline{\mathrm{X}}-\overline{\mathrm{A}}$ as shown in Fig. 4e. The measured position agrees with the calculated band touching point marked as the blue dot in Fig. 4d. This particular touching point of band projection is the cut position of in-plane nodal chain at the Brillouin zone boundary $(\overline{\mathrm{X}}-\overline{\mathrm{A}})$ as illustrated in Fig. 4b. Surface modes can also be found for the side surface configuration, which are indicated with the orange curve in Fig. $4 \mathrm{~d}$ and white dotted curve in Fig. 4e. Meanwhile, indications of the nodal ring degeneracies can also be found in the projected band spectra on side surface. The nodal ring is 

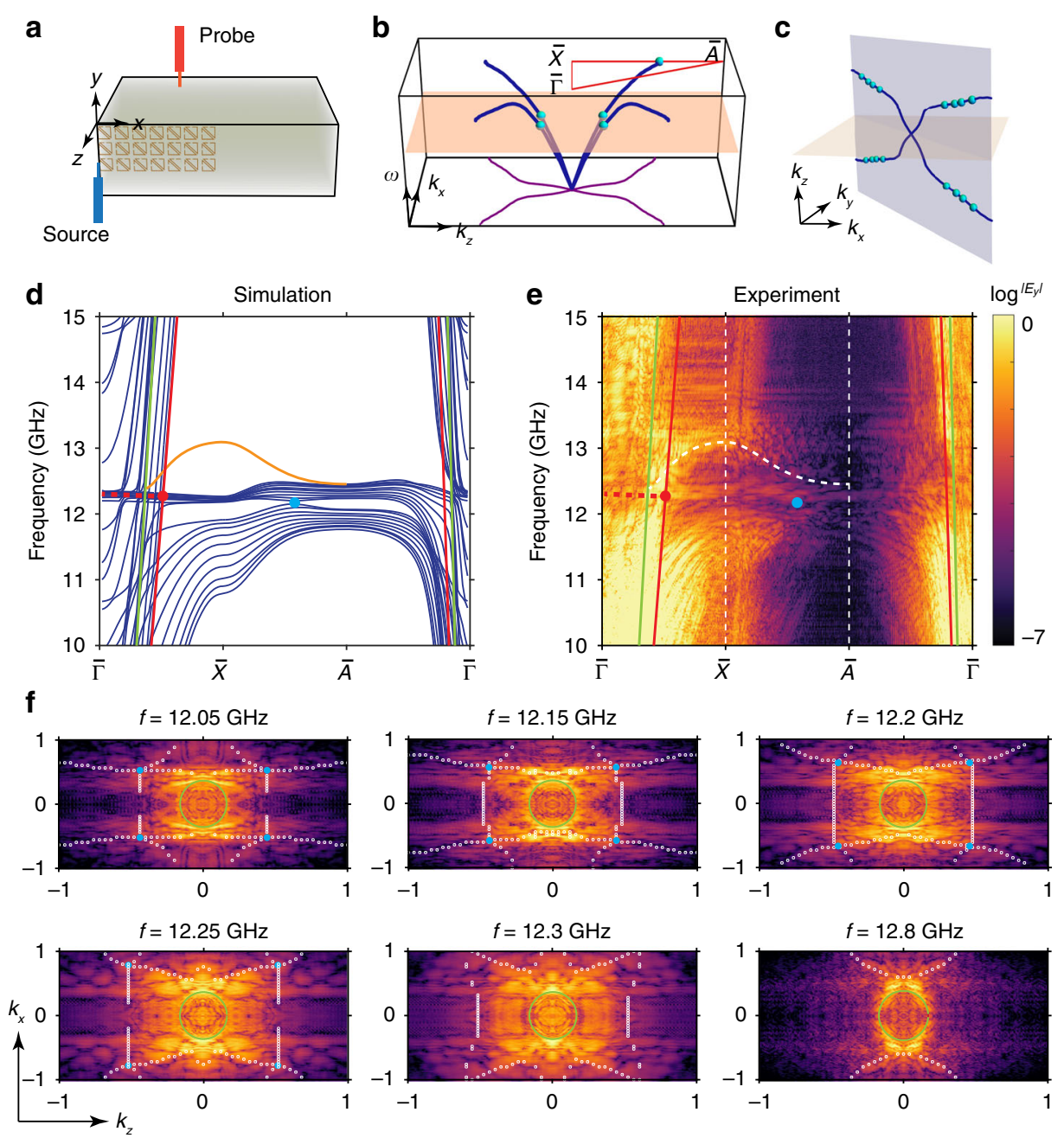

Fig. 4 Experimental mapping of in-plane nodal chain within bi-anisotropic metamaterials. a Schematic of bi-anisotropic metamaterials for side surface (xz plane) scanning. Source and probe antennas are indicated in blue and red, respectively. $\mathbf{b}$ Calculated in-plane nodal chain (blue curves) dispersion, and its projection onto the $k_{x}-k_{z}$ plane as the in-plane nodal chain (purple curves). Cyan dots show the degenerate points on one particular equi-frequency plane. Red triangle shows the path for the band spectra shown in $\mathbf{d}$, e. c In-plane nodal chain in momentum space, blue lines are calculated results and cyan dots are from measurement results of f. d, e Calculated and experimentally mapped band spectra along $\bar{\Gamma}-\bar{X}-\bar{A}$

$-\bar{\Gamma}$. For both simulation and experimental results, the blue dot marks the point where the nodal chain meets the Brillouin zone boundary on the $\bar{X}-\bar{A}$ line. Surface mode is indicated with orange curve in $\mathbf{d}$ and white dashed curve in $\mathbf{e}$. Nodal ring projection is indicated with red dashed line. Light cone for air and effective substrate $\left(\varepsilon_{b} \approx 1.95\right)$ are shown with green and red solid lines, respectively. $\mathbf{f}$ Experimentally mapped EFCs for different frequencies. Calculated EFCs are marked with white dots. Blue dots indicate the nodal points at the corresponding frequencies. Green rings are the EFCs of light cone for air

located at the $k_{z}=0$ plane and projected onto the $\bar{\Gamma}-\overline{\mathrm{X}}$ line of Fig. $4 \mathrm{~b}$ for the side surface configuration. In the $\bar{\Gamma}$ $\overline{\mathrm{X}}$ part of Fig. 4d, e, longitudinal flat band projections are indicated with red dashed lines, and the transverse mode for $k_{z}=k_{y}=0$ (also the light cone for substrate) is plot as red solid line. The degeneracy point between them is indicated as red dot, which is the nodal ring degeneracy projection along $\bar{\Gamma}-\overline{\mathrm{X}}$. We further show the mapped EFCs in Fig. 4f with respect to several frequencies from $f=$ $12.05 \mathrm{GHz}$ to $f=12.3 \mathrm{GHz}$. At each frequency, the boundaries of projected bands from the calculations are shown as white dots. Blue dots are the EFCs-cut nodal points explained in Fig. 4b and shown together in Fig. $4 \mathrm{c}$ as cyan dots. At a higher frequency of $f=12.8 \mathrm{GHz}$, the EFCs of surface mode is measured and indicated with white dashed lines in the last panel of Fig. $4 \mathrm{f}$ (more discussions of surface states in supplementary information 7).

In a different experimental configuration, we measured the top surface (xy plane) field distributions of the layered metamaterials in Fig. 5a. We used a sample containing $100 \times 100$ unit cells in the xy plane and 10 layers in the $\mathrm{z}$ direction. The surface Brillouin zone is shown in Fig. 5b, 
a

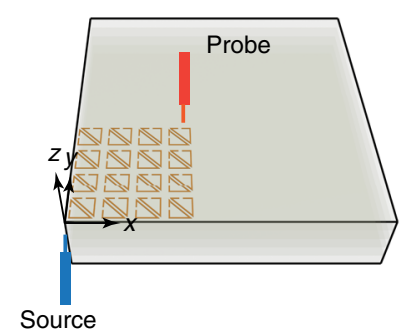

d

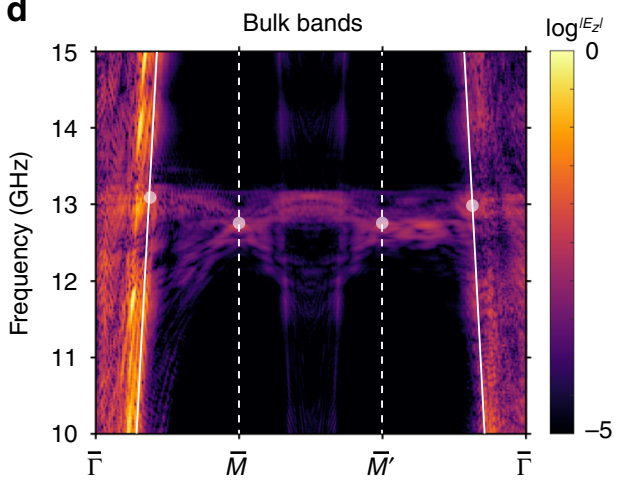

f

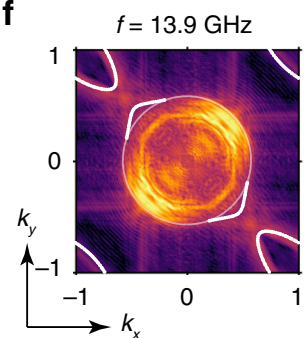

$f=14.1 \mathrm{GHz}$

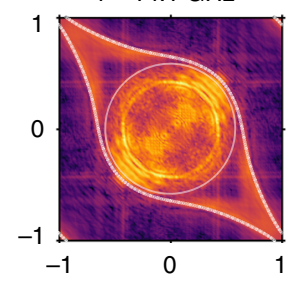

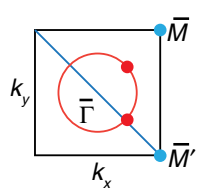

b

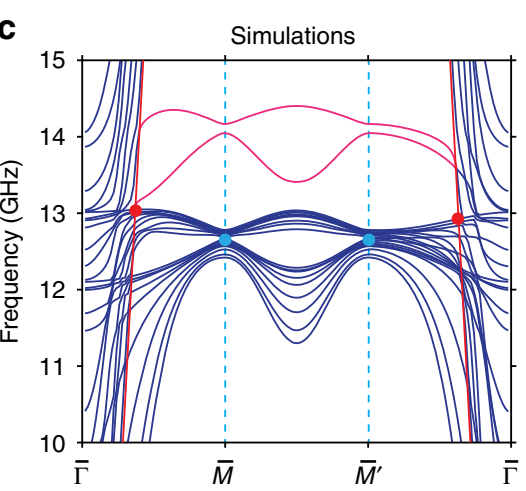

e

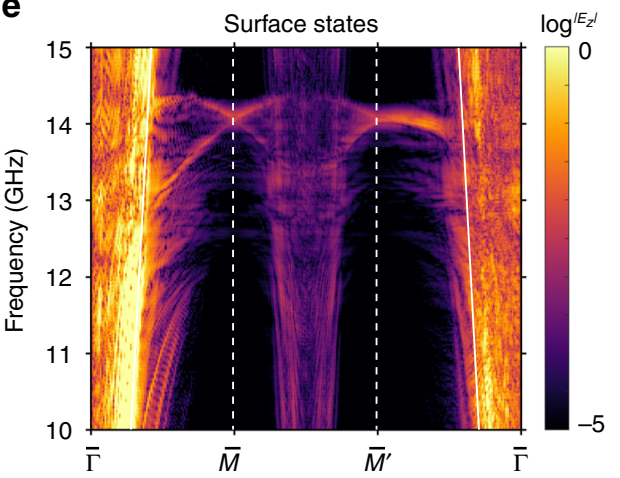

$f=14.2 \mathrm{GHz}$

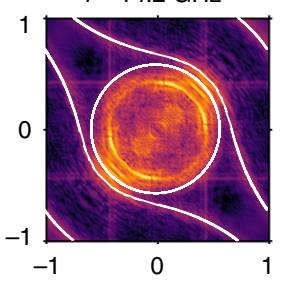

$f=14.3 \mathrm{GHz}$

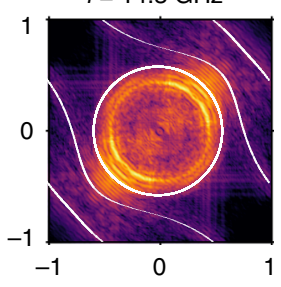

Fig. 5 Experimental measurements of nodal lines and surface modes at the top surface. a Schematic of the experimental measurement configuration on the top surface (xy plane). The source and probe antennas are colored in blue and red, respectively. $\mathbf{b}$ Surface Brillouin zone of the top surface. Blue line indicates the projection of in-plane nodal chain and red circle is the nodal ring projection. c Calculated projected band spectra for the top surface. Surface modes are indicated with red color. Blue (Red) dots indicate the degenerate points on the in-plane nodal chain (nodal ring), as also illustrated in $\mathbf{b}$. Substrate light cone is shown as red solid lines $\left(\varepsilon_{b} \approx 1.8\right)$. It should be noted that the slight difference of effective permittivity from above measurement is due to the sample compactness in two configurations. $\mathbf{d}$, e Measured bulk bands and surface states on the top surface, respectively. Degeneracy positions of nodal chain (ring) are indicated with white dots in $\mathbf{d}$. Substrate light cones are shown as white solid lines. $\mathbf{f}$ Measured and calculated (white lines) EFCs of surface states at the frequencies of $f=[13.9,14.1,14.2,14.3] \mathrm{GHz}$. EFCs for substrate light cone are shown as white circles

with the blue line indicating the projected in-plane nodal chain and the red circle marking the nodal ring. The projected band dispersion along the $\mathrm{z}$ direction is shown in Fig. $5 \mathrm{c}$, where the projected bulk bands have degeneracies at $\bar{M}$ and $\overline{\mathrm{M}}^{\prime}$ points around $f=12.5 \mathrm{GHz}$ (indicated with blue dots). These degeneracies at $\bar{M} / \bar{M}^{\prime}$ originate from the in-plane nodal chain as explained and marked in Fig. 5b. We note that the projected bulk bands along $\bar{\Gamma}-\overline{\mathrm{M}}^{\prime}$ possess no energy gap around $12.5 \mathrm{GHz}$ in contrast to the bands along $\bar{\Gamma}-\bar{M}$, which is a result of the in-plane nodal chain projection long $-45^{\circ}$ diagonal direction (blue line in Fig. 5b). Additional degeneracy positions of projected bands can be seen along $\bar{\Gamma}-\overline{\mathrm{M}} / \overline{\mathrm{M}}^{\prime}$ as red dots. These crossings are between the longitude modes (nearly flat bands) and transverse modes (close to light cone in red), which marks the nodal ring degeneracies. Surface modes are found above the bulk bands, as indicated with red lines.

By tuning the source antenna position along the $\mathrm{z}$ direction, the bulk and surface states can be selectively 
excited as demanded. During the measurement of bulk bands, a thin layer of dielectric is used to cover the top of the layered metamaterials, and the modified boundary condition eliminates the high frequency surface modes. We show the measured bulk bands in Fig. 5d. The degeneracies at $\bar{M}$ and $\bar{M}^{\prime}$ points are experimentally observed for the in-plane nodal chain and indicated with white dots. The crossings between transverse and longitude modes are also observed and marked with white dots near the light cone (white straight lines), which can be identified as the nodal ring projection in Fig. 5b. Removing the dielectric layer covering the top, the surface modes can be observed. The measured surface state bands are shown in Fig. 5e, and surface modes are found at higher frequencies as in good accordance with the calculated results of Fig. 5c. We also show the EFCs of surface modes with respect to different frequencies in Fig. 5f, where calculated (white lines) and measured results can also be found with good accordance.

\section{Discussion}

The photonic in-plane nodal chain realized in our system is protected by mirror symmetry and electromagnetic intrinsic degeneracy at zero frequency. The latter is robust as long as no plasmon resonance is introduced to gap the $\Gamma$ point, such as introducing metallic wires in metamaterials. The nodal link constructed between three consecutive bands can be characterized with non-Abelian topological charges, which provide extra protections to the nodal structure at the $C_{2} T$ - invariant plane as illustrated in Fig. 2e, f.

The in-plane nodal chain (even the nodal link) should widely exist in photonic systems, and the bi-anisotropic metamaterials studied here serve as a clean and simple model with the necessary and sufficient symmetry conditions to manifest the in-plane nodal chain and nodal link (supplementary information 8, 9). Breaking the mirror symmetry would transform the nodal lines into Weyl points (supplementary information 10). Our metamaterial system thus provides a platform for studying topological phase transitions. The novel structure of linked in-plane nodal chain and nodal ring in our demonstrated system could also find applications in designing novel functioning photonic devices.

\section{Materials and methods}

Simulations are carried out with CST microwave studio and Comsol Multiphysics. The sample is fabricated with Printed Circuit Board techniques. For top surface measurement, a 10-layer sample with $100 \times 100$ unit cells was fabricated, the whole sample size is $45 \mathrm{~cm}$ by $45 \mathrm{~cm}$ by $4 \mathrm{~cm}$. For the side surface measurement, $80 \times 100$ unit cells are configurated, and the thickness of sample is $4.5 \mathrm{~cm}$ (10 unit cells). The experimental measurements are conducted with microwave near field scanning system, where a vector network analyzer (VNA) is used to connect both the source and probe antennas. The field information is Fourier transformed to achieve the projected bands and EFCs.

\section{Acknowledgements}

This work is supported by the Research Grants Council of Hong Kong, China (AoE/P-02/12, 16304717, 16310420) and the Hong Kong Scholars Program (XJ2019007). W.-J.C. is supported by the National Natural Science Foundation of China (Grant no. 11874435). J.H. is supported by the National Natural Science Foundation of China (Grant no. 62025504, 61935015). S.Z.

acknowledges support from the ERC Consolidator Grant (TOPOLOGICAL), the Royal Society, and the Wolfson Foundation.

\section{Author details}

${ }^{1}$ Department of Physics, Hong Kong University of Science and Technology, Hong Kong, China. ${ }^{2}$ College of Advanced Interdisciplinary Studies, National University of Defense Technology, Changsha, China. ${ }^{3}$ Institute for Advanced Study, Hong Kong University of Science and Technology, Hong Kong, China. ${ }^{4}$ Key Laboratory for Micro-Nano Optoelectronic Devices of Ministry of Education, School of Physics and Electronics, Hunan University, Changsha, China. ${ }^{5}$ Institute of Solid State Physics and Department of Physics, Shanxi Datong University, Datong, China. ${ }^{6}$ School of Physics \& State Key Laboratory of Optoelectronic Materials and Technologies, Sun Yat-Sen University,

Guangzhou, China. ${ }^{7}$ Center for Terahertz Waves and College of Precision Instrument and Optoelectronics Engineering, Tianjin University and the Key Laboratory of Optoelectronics Information and Technology (Ministry of Education), Tianjin, China. ${ }^{8}$ School of Physics \& Astronomy, University of Birmingham, Birmingham, UK

\section{Author contributions}

D.W., B.Y., S.Z., and C.T.C. initiated the project and designed the experiment. D.W., Q.G., W.-J.C., and J.H. fabricated samples. D.W., Q.G., X.S. L.X., and W.-J.C. carried out the measurements. D.W., B.Y., R.-Y.Z., X.S., L.X., W.-J.C., J.H., S.Z., and C.T.C. analyzed data. D.W. and Q.G. performed simulations. D.W., B.Y., R.-Y.Z., J.H., S.Z., and C.T.C. provided the theoretical explanations. J.H., S.Z., and C.T.C. supervised the project. All authors discussed the results and contributed to the final manuscript.

Conflict of interest

The authors declare no competing interest.

Supplementary information The online version contains supplementary material available at https://doi.org/10.1038/s41377-021-00523-8.

Received: 24 November 2020 Revised: 15 March 2021 Accepted: 30 March 2021

Published online: 15 April 2021

\footnotetext{
References

1. Ozawa, T. et al. Topological photonics. Rev. Mod. Phys. 91, 015006 (2019).

2. Kim, M., Jacob, Z. \& Rho, J. Recent advances in $2 D, 3 D$ and higher-order topological photonics. Light.: Sci. Appl. 9, 130 (2020).

3. Bahari, B. et al. Nonreciprocal lasing in topological cavities of arbitrary geometries. Science 358, 636-640 (2017).

4. Harari, G. et al. Topological insulator laser: theory. Science 359, eaar4003 (2018).

5. Bandres, M. A. et al. Topological insulator laser: experiments. Science $\mathbf{3 5 9}$, eaar4005 (2018)

6. Zeng, Y. Q. et al. Electrically pumped topological laser with valley edge modes. Nature 578, 246-250 (2020)

7. Yasutomo, O. et al. Active topological photonics. Nanophotonics 9, 547-567 (2020).

8. Rechtsman, M. C. et al. Photonic Floquet topological insulators. Nature 496, 196-200 (2013).
} 
9. Li, M. Y. et al. Higher-order topological states in photonic kagome crystals with long-range interactions. Nat. Photonics 14, 89-94 (2020).

10. Haldane, F. D. M. \& Raghu, S. Possible realization of directional optical waveguides in photonic crystals with broken time-reversal symmetry. Phys. Rev. Lett. 100, 013904 (2008).

11. Raghu, S. \& Haldane, F. D. M. Analogs of quantum-Hall-effect edge states in photonic crystals. Phys. Rev. A 78, 033834 (2008).

12. Wang, Z. et al. Reflection-free one-way edge modes in a gyromagnetic photonic crystal. Phys. Rev. Lett. 100, 013905 (2008).

13. Wang, Z. et al. Observation of unidirectional backscattering-immune topological electromagnetic states. Nature 461, 772-775 (2009).

14. Hafezi, M. et al. Robust optical delay lines with topological protection. Nat. Phys. 7, 907-912 (2011).

15. Lu, L. et al. Weyl points and line nodes in gyroid photonic crystals. Nat. Photonics 7, 294-299 (2013).

16. Khanikaev, A. B. et al. Photonic topological insulators. Nat. Mater. 12, 233-239 (2013).

17. Lu, L., Joannopoulos, J. D. \& Soljačić, M. Topological photonics. Nat. Photonics 8, 821-829 (2014).

18. Chen, W. J. et al. Experimental realization of photonic topological insulator in a uniaxial metacrystal waveguide. Nat. Commun. 5, 5782 (2014).

19. Lu, L. et al. Experimental observation of Weyl points. Science 349, 622-624 (2015).

20. Gao, W. L. et al. Topological photonic phase in chiral hyperbolic metamaterials. Phys. Rev. Lett. 114, 037402 (2015).

21. Xiao, M., Lin, Q. \& Fan, S. H. Hyperbolic weyl point in reciprocal chiral metamaterials. Phys. Rev. Lett. 117, 057401 (2016).

22. Chen, W. J., Xiao, M. \& Chan, C. T. Photonic crystals possessing multiple Weyl points and the experimental observation of robust surface states. Nat. Commun. 7, 13038 (2016).

23. Yang, B. et al. Direct observation of topological surface-state arcs in photonic metamaterials. Nat. Commun. 8, 97 (2017).

24. Guo, Q. H. et al. Three dimensional photonic dirac points in metamaterials. Phys. Rev. Lett. 119, 213901 (2017).

25. Dong, J. W. et al. Valley photonic crystals for control of spin and topology. Nat. Mater. 16, 298-302 (2017).

26. Yang, B. et al. Ideal Weyl points and helicoid surface states in artificial photonic crystal structures. Science 359, 1013-1016 (2018).

27. Guo, Q. H. et al. Observation of three-dimensional photonic dirac points and spin-polarized surface arcs. Phys. Rev. Lett. 122, 203903 (2019).

28. Wang, D. Y. et al. Photonic Weyl points due to broken time-reversal symmetry in magnetized semiconductor. Nat. Phys. 15, 1150-1155 (2019).

29. Hasan, M. Z. \& Kane, C. L. Colloquium: topological insulators. Rev. Mod. Phys. 82 3045-3067 (2010).

30. Qi, X. L. \& Zhang, S. C. Topological insulators and superconductors. Rev. Mod. Phys. 83, 1057-1110 (2011).

31. Watanabe, H. \& Lu, L. Space group theory of photonic bands. Phys. Rev. Lett. 121, 263903 (2018)

32. Chen, W. J. et al. Metamaterials with index ellipsoids at arbitrary k-points. Nat. Commun. 9, 2086 (2018)

33. Xiong, Z. F. et al. Hidden-symmetry-enforced nexus points of nodal lines in layer-stacked dielectric photonic crystals. Light.: Sci. Appl. 9, 176 (2020).

34. Bzdušek, T. et al. Nodal-chain metals. Nature 538, 75-78 (2016).

35. Liu, Z. H. et al. Experimental observation of dirac nodal links in centrosymmetric semimetal TiB2. Phys. Rev. X 8, 031044 (2018).
36. Lou, R. et al. Experimental observation of bulk nodal lines and electronic surface states in ZrB2. npj Quantum Mater. 3, 43 (2018).

37. Yan, Q. H. et al. Experimental discovery of nodal chains. Nat. Phys. 14, 461-464 (2018).

38. Gong, C. et al. Symmorphic intersecting nodal rings in semiconducting layers. Phys. Rev. Lett. 120, 106403 (2018).

39. Fang, C. et al. Topological nodal line semimetals with and without spin-orbital coupling. Phys. Rev. B 92, 081201 (2015).

40. Yu, R. et al. Topological node-line semimetal and dirac semimetal state in antiperovskite Cu3PdN. Phys. Rev. Lett. 115, 036807 (2015).

41. Weng, H. M. et al. Topological node-line semimetal in three-dimensional graphene networks. Phys. Rev. B 92, 045108 (2015).

42. Bi, R. et al. Nodal-knot semimetals. Phys. Rev. B 96, 201305 (2017)

43. Yu, R. et al. From nodal chain semimetal to weyl semimetal in HfC. Phys. Rev. Lett. 119, 036401 (2017).

44. Ezawa, M. Topological semimetals carrying arbitrary Hopf numbers: fermi surface topologies of a Hopf link, Solomon's knot, trefoil knot, and other linked nodal varieties. Phys. Rev. B 96, 041202 (2017).

45. Kawakami, T. \& Hu, X. Symmetry-guaranteed nodal-line semimetals in an fcc lattice. Phys. Rev. B 96, 235307 (2017).

46. Bzdušek, T. \& Sigrist, M. Robust doubly charged nodal lines and nodal surfaces in centrosymmetric systems. Phys. Rev. B 96, 155105 (2017).

47. Chen, W., Lu, H. Z. \& Hou, J. M. Topological semimetals with a double-helix nodal link. Phys. Rev. B 96, 041102 (2017).

48. Yan, Z. B. et al. Nodal-link semimetals. Phys. Rev. B 96, 041103 (2017).

49. Chang, P. Y. \& Yee, C. H. Weyl-link semimetals. Phys. Rev. B 96, 081114 (2017).

50. Ahn, J. et al. Band topology and linking structure of nodal line semimetals with Z2 monopole charges. Phys. Rev. Lett. 121, 106403 (2018).

51. Sun, X. Q., Zhang, S. C. \& Bzdušek, T. Conversion rules for weyl points and nodal lines in topological media. Phys. Rev. Lett. 121, 106402 (2018).

52. Xie, Y. et al. Hopf-chain networks evolved from triple points. Phys. Rev. B 99, 165147 (2019).

53. Yang, Z. S. et al. Jones polynomial and knot transitions in hermitian and nonhermitian topological semimetals. Phys. Rev. Lett. 124, 186402 (2020).

54. Wu, Q. S., Soluyanov, A. A. \& Bzdušek, T. Non-Abelian band topology in noninteracting metals. Science 365, 1273-1277 (2019).

55. Lenggenhager, P. M. et al. From triple-point materials to multiband nodal links. Phys. Rev. B 103, L121101 (2021).

56. Tiwari, A. \& Bzdušek, T. Non-Abelian topology of nodal-line rings in PTsymmetric systems. Phys. Rev. B 101, 195130 (2020).

57. Bouhon, A. et al. Non-Abelian reciprocal braiding of Weyl points and its manifestation in ZrTe. Nat. Phys. 16, 1137-1143 (2020).

58. Yang, E. C. et al. Observation of Non-Abelian nodal links in photonics. Phys. Rev Lett. 125, 033901 (2020)

59. Guo, Q. H. et al. Experimental observation of non-Abelian topological charges and bulk-edge correspondence. arXiv preprint arXiv: 2008.06100 (2020).

60. Kléman, M., Michel, L. \& Toulouse, G. Classification of topologically stable defects in ordered media. J. de. Phys. Lett. 38, 195-197 (1977).

61. Mermin, N. D. The topological theory of defects in ordered media. Rev. Mod. Phys. 51, 591-648 (1979).

62. Gao, W. L. et al. Experimental observation of photonic nodal line degeneracies in metacrystals. Nat. Commun. 9, 950 (2018).

63. Xia, L. B. et al. Obsenvation of hourglass nodal lines in photonics. Phys. Rev. Lett. 122, 103903 (2019). 